\title{
A New Legendre Wavelets Neural Network Algorithm for Evaluating English Practice Base Teaching
}

\author{
Xuehui Pei ${ }^{1,}$ a, Guixin Pei ${ }^{2, b}$ \\ ${ }^{1}$ Department of E-commerce and Management, Jiangxi Vocational and Technical College of \\ Information Application, Nanchang 330000, China; \\ ${ }^{2}$ Meteorology Bureau of Qian'an City, Qian'an 064400, China \\ a407706483@qq.com, bliyi7611@163.com
}

\begin{abstract}
Keywords: Legendre wavelets, BP neural network, teaching evaluation, English practice base teaching.
\end{abstract}

\begin{abstract}
Practice base teaching evaluation plays an important role in university teaching management and is a hotspot research field for researchers related. The paper takes university English practice base teaching for research sample, and presents a new Legendre wavelets neural network algorithm to evaluate practice base teaching. Firstly, an indicator system of university English practice base teaching evaluation is designed through analyzing the aspects of teaching preparation, specific teaching, teaching administration and teaching effects. Secondly, Legendre wavelets are used to improve BP neural network algorithm to overcome its shortages of low convergence in calculation based on analysis of BP working principle. Thirdly, taking sample research data from three universities, the experimental results indicates that the presented Legendre wavelets neural network algorithm can be used in evaluating English practice base teaching for different universities and guarantee the evaluation validity and effectiveness.
\end{abstract}

\section{Introduction}

English practice base plays great role in training students' language abilities in English listening, speaking, reading, writing, translation and serving the first line. And it can facilitate the realization of talent training targets and embody the school running direction and characteristics of English language teaching. Base teaching evaluation system, taking English practice base teaching as evaluation object, carries out special evaluation on the training base teaching. Through the comprehensive evaluation of university base training teaching, training status of university base training education in talent training work of school, students' specific practice training ability and teaching parts needing perfection can be reflected roundly, complementary with in-school theoretical teaching and in-school practice teaching, playing an important guiding role in the construction of base training teaching in universities. Therefore, to launch the comprehensive evaluation study on university base teaching including the establishment of evaluation indicator system and evaluation method study has significant theoretical and practical value, also one of the research hotspots in the industry.

\section{Literature Review of Evaluation Methods}

Up to now, mathematical models adopted by evaluation of practice base teaching performance mainly include the following categories. (1) Analytic hierarchy process is a good method for quantitative evaluation via quantitative method, having the functions of establishing the ideal weight structure of evaluated object value and analyzing the weight structure of actually-built value by evaluated object; however, the method has strong limitations and subjectivity, with large personal error, not suitable for complicated system with lots of evaluation indicators[1]. (2)Fuzzy comprehensive evaluation is a method carrying out comprehensive evaluation and decision on system through fuzzy set theory, the greatest advantage of which is that it works well on system evaluation of multi-factor and multi-level complicated problems. However, the membership of fuzzy evaluation method as well 
as the definition and calculation of membership function are too absolute, difficult to reflect the dynamics and intermediate transitivity of evaluation indicators of innovation education performance[2]; (3)BP neural network evaluation method makes use of its strong capability in processing nonlinear problems to carry out evaluation of innovation education performance; the method has advantages like self-learning, strong fault tolerance and adaptability; however, the algorithm is easy to be trapped into defects like local minimum, over-learning, strong operation specialization[3].

Based on BPNN, Legendre wavelets neural network is being constructed with Legendre wavelets in this paper. In so doing, not only the problem of convergence speed has been solved, but also the simplicity of the model structure and the accuracy of the teaching performance evaluation are ensured.

\section{Evaluation Indicator System Construction}

On the basis of referring to references $[4,5,6]$, experts consultation and practice survey, the paper designs a set of evaluation indicator system of university English practice teaching according to base teaching preparation, specific process, management and effect, which includes 4 first-class indicators that are teaching preparation, specific teaching, specific administration, and teaching effects. And teaching preparation includes 4 second-class indicators which are teachers(including third-class indicators :teachers' quality, teachers' structure),teaching documents(including third-class indicators : teaching syllabus, teaching plan and teaching instruction), teaching facilities(including third-class indicators : teaching venue, teaching equipment, teaching safety and skill test point) and practice discipline(including third-class indicators: teaching discipline); Specific teaching includes 3 second-class indicators which are teaching guidance(including third-class indicators : mentoring ability and instructional method), teaching order(including third-class indicators : teaching site and students' feedback) and teaching supervision(including third-class indicators : implementation of teaching contents and implementation of teaching progress); Specific administration includes 3 second-class indicators which are organizational management(including third-class indicators : organization in place and task arrangement), system management(including third-class indicators : system soundness and system implementation) and management effect(including third-class indicators : faculty responsibility and diary for instruction); Teaching effects includes 3 second-class indicators which are skill mastery(including third-class indicators : comprehensive English quality and English applictaion ability),teaching achievement(including third-class indicators : task completion quality and social evaluation) and teaching experience(including third-class indicators : teaching report and teaching summary).

\section{Evaluation Algorithm Design}

\subsection{Working Principle of BP Neural Network Algorithm}

Up till now, hundreds of artificial neural network models are put forward from different views of research, among which multi-hierarchy feed forward error back propagation BP neural network is the most-widely used network model in actual research. Basic three-layer BP neural network structure is shown as Figure 1[3].

From the picture we can see that three- layer BP neural network is mainly comprised of input layer, hidden layer and output layer. Adjustable weight $\omega$ connects the layers. There can be several hidden layers, forming multi-layer BP neural network. The input of BP neural network is recorded as $x_{i}(k)$, the actual output of network is recorded as $y_{j}(k)$, the ideal output of network is recorded as $Y_{i}(k)$, the subscripts $i, j$ indicate the nodes of input layer of network respectively, and $k$ is the running iterations of BP neural network. Its approximation error is defined as Formula 1 in which $L$ is the quantity of output layer nodes; in this way, the function characteristic of BP neural network can be described as Formula 2[3]. 


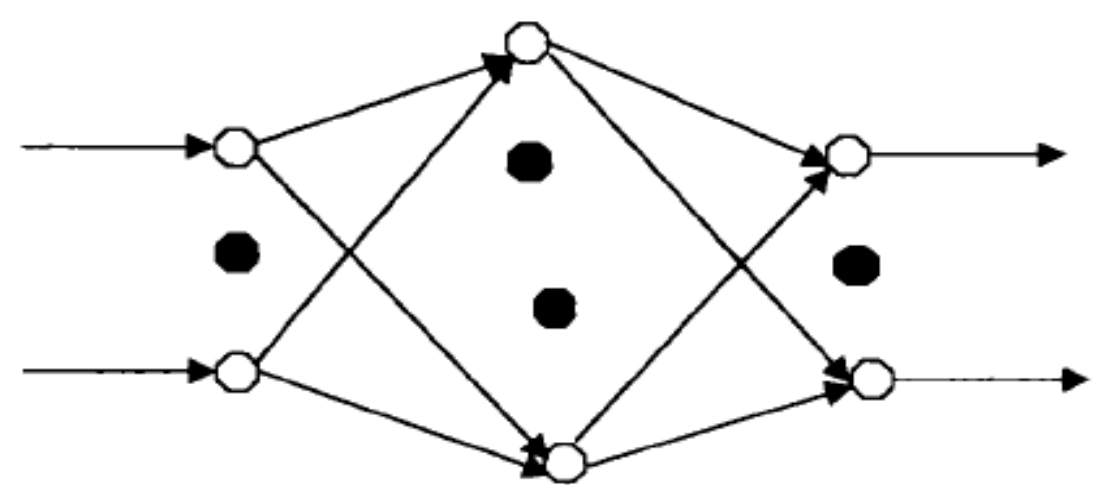

Fig. 1 Basic structure of BP neural network

$$
\begin{aligned}
& E=\frac{1}{2} \sum_{j=1}^{L}\left(Y_{j}(k)-\gamma_{j}(k)\right)^{2} \\
& \gamma_{j}(k)=f\left(x_{i}(k), \omega\right)
\end{aligned}
$$

\subsection{Legendre Wavelets Neural Network Design}

Wavelets can provide multi-resolution proximity for function differentiation as well as localization of space and frequency. Therefore, wavelets neural network based on wavelets analysis theory is more adaptable to learn locally non-linear and rapidly changing functions. Legendre wavelets is Formula3, in which $m$, the order of Legendre polynomial and $t$, the time, are defined in the interval[0,1) to satisfy Formula 4. In Formula $4 L_{m}(t)$ is the Legendre polynomial, in which $L_{0}(t)=1 、 L_{1}(t)=t$ and the others satisfy the Recursion Formula 5. It can be proved that for different values of $n$, Legendre wavelets remain orthonormal [7].

$$
\begin{aligned}
& \psi_{n m}(t)=\psi(k, \hat{n}, m, t), \quad k=2,3 \ldots, \quad \hat{n}=2 n-1, \quad n=1,2 \ldots 2^{k-1} \\
& \psi_{n m}(t)= \begin{cases}{\left[m+\frac{1}{2}\right]^{1 / 2} 2 k / 2 L_{m}\left(2^{k} t-\hat{n}\right),} & \frac{\hat{n}-1}{2^{k}} \leq t \leq \frac{n+1}{2^{k}} \\
0 & \text { others }\end{cases} \\
& L_{m+1}(t)=\frac{2 m+1}{m+1} t L_{m}(t)-\frac{m}{m+1} L_{m-1}(t)
\end{aligned}
$$

From Formula 5, it can be known that a function $f(t)$ defined in the interval $[0,1)$ can be approximated to be Formula 6, in which $C$ and $\psi(t)$ are Formula 7 and Formula 8 respectively.

$$
\begin{aligned}
& f(t) \approx \sum_{n=1}^{2 k-1} \sum_{m=0}^{M-1} \rho_{n m} \psi_{n m}(t)=C^{T} \psi(t) \\
& C=\left[c_{10}, c_{11} \ldots, c_{1 M-1}, c_{20} \ldots c_{2 M-1} \ldots c_{2^{k-1} 0} \ldots c_{2^{k-1} M-1}\right. \\
& \psi(t)=\left[\psi_{10}(t), \psi_{11}(t), \ldots \psi_{1 M-1}(t) \ldots \ldots . \psi_{2^{k-1} M-1}(t)\right.
\end{aligned}
$$

By setting as the activation function of neural network, a Legendre wavelets neural network can be constructed through Formula 8 with a structure as follows:

(1) Input layer: to input digitalized original signals.

(2) Preprocessing layer: to divide the digitalized original signals inputted into $2^{k-1}$ groups, which will enter the corresponding Legendre wavelets basic function to get training.

(3) Hidden layer: divided into $2^{k-1}$ group nodes with each having $M$ Legendre wavelets basic functions to receive signals after preprocessing respectively. The weight for the hidden layer nodes are the proximity of Legendre wavelets coefficients.

(4) Output layer: to receive the output of the hidden layer. The output layer is linear nodes which are added to get the result. 


\section{Experiment Confirmation and Conclusion}

Experimental data come from database of Jiangxi Normal University, and Nanchang University and Jiangxi University of Finance and Economics. Relevant data of 3000 learner of each university are selected as the basis for data training and experimental verification in the paper, totally 9000 learns' data for study data that come from practical investigation and visit of different students. As for the performance of the presented algorithm, this thesis also realizes the application of the ordinary BP neural network [3] and fuzzy comprehensive evaluation algorithm [2], evaluation performance of different algorithms is shown in Table 1. In table 1 evaluation results of training effects of different students are selected and compared with artificial evaluation to calculate the evaluation accuracy. And the calculation platform as follows: hardware is Dell Poweredge R710, in which processor is E5506, memory 2G, hard disk 160G; software platform is Windows XP operating system, C programming language environment.

Table 1 Evaluation Performance Comparison of Different Algorithms

\begin{tabular}{cccc}
\hline & Algorithm in the paper & $\begin{array}{c}\text { Ordinary BP } \\
\text { neural network }\end{array}$ & $\begin{array}{c}\text { Fuzzy comprehensive } \\
\text { evaluation algorithm }\end{array}$ \\
\hline Evaluation Accuracy & $95.48 \%$ & $84.63 \%$ & $80.64 \%$ \\
Time Consuming (S) & 12 & 762 & 13 \\
\hline
\end{tabular}

From table 1, it can show that, compared with ordinary BP neural network algorithm and fuzzy comprehensive evaluation algorithm, the algorithm presented in the paper has favorable practicability and evaluation accuracy when used in evaluating English base teaching.

\section{References}

[1]. Tomas J. H., Thaile Z. W. Analyzing the Complex System Evaluation Based on Analytic hierarchy process. Journal of Computer Application. Vol. 10 (2015) No. 10, p. 1301-1310.

[2]. Ya Y. S., Janst S. H. Fuzzy Quality Attributes for Evaluating for the University Practice Base Teaching. Journal of Information Engineering. Vol. 14 (2013) No. 12, p. 931-936.

[3]. Lee Y. J. Construction of BPMN-based Business Process Model Base. International Journal of Intelligent Information Processing. Vol. 21 (2015) No. 1, p. 32-46.

[4]. Wang S. H. Diversified University Teaching Performance Assessment. Journal of Education Management. Vol. 15 (2014) No. 11, p. 231-236.

[5]. Wang B., Ye K. L. A Probe into the Construction of Base Training in Normal Institutes_-A Case Study Concerning the Construction of Martial Arts training Base in Neijiang Normal University. Journal of Neijiang Normal University. Vol. 24 (2016) No. 3, p. 103-107.

[6]. Ren Y. H. Reconstruction and Reflection of University Training Base Education. Journal of Education Management. Vol. 15 (2014) No. 1, p. 24-31.

[7]. Kadhi N. E., Hadjar K. S. A Mobile Agent and Artificial Neural Networks for Intrusion Detection. Journal of Software. Vol. 7 (2012) No. 1, p. 156-167. 